\title{
Miscible Displacement in Porous Media in the Presence of Nanocatalytic Reaction
}

\author{
Nasser Sabet, Seyed Mostafa Jafari Raad, Hassan Hassanzadeh, Jalal Abedi \\ Department of Chemical and Petroleum Engineering, Schulich School of Engineering, University of Calgary \\ Calgary, Alberta, Canada, T2N 1N4 \\ nsabet@ucalgary.ca; seyedmostafa.jafarir@ucalgary.ca; hhassanz@ucalgary.ca; jabedi@ucalgary.ca
}

\section{Extended Abstract}

In the past decade, diverse application of nanocatalysts in different areas of science and engineering such as soil remediation [1] and heavy oil and bitumen hydrocarbon upgrading [2] has drawn many attentions. In these applications, nanocatalysts are used ex-situ or in-situ in a carrier phase to turn a target substance into more desirable or less harmful products. For instance, for the in-situ upgrading of heavy oil, hot vacuum gas-oil (VGO) containing multi-metallic nanocatalysts was injected into a sand pack saturated with bitumen at high temperatures [2]. As a result of the cracking reaction in the presence of nanocatalysts, $10 \%$ increase in bitumen production was observed. Despite many experimental studies on the utilization of nanocatalysts in the literature, there is a lack of theoretical understanding about the mixing of fluids in the presence of nanocatalytic reaction and the effectiveness of the application of nanocatalysts in porous media.

In this study, we aim to investigate the mixing and conversion of materials in the presence of nanocatalysts. We define the governing equations for the transport of nanocatalysts taking into account their deposition in porous media and the nanocatalytic reaction. The governing equations are made dimensionless using the diffusive scaling. Among the different techniques for the linear stability analysis [3], [4], we employ the Quasi-Steady-State approximation (QSSA) to characterize the effect of different parameters. In this regard, we solve the linearized form of equations both analytically (for $t=0^{+}$) and numerically (for $\mathrm{t}>0$ ). Based on the numerical solution, we plot the maximum instantaneous growth rate versus time to track the behavior of interfacial instabilities. Moreover, we carry out nonlinear numerical simulations based on the Hartly-based pseudo-spectral technique [5], [6] to validate the results of the linear stability analysis and also to characterize the nanocatalysts efficiency in terms of the degree of mixing and the global production rate of the product.

The results show that the increase in the nanocatalyst log mobility ratio results in less interfacial instability, while the increase in the reaction rate leads to a more unstable interface. Regarding the effect of the deposition rate of nanocatalysts in porous media, our results reveal that two competing mechanisms control the mixing of fluids: (i) deposition-reaction effect (DRE), and (ii) deposition-mobility effect (DME). When DRE is the dominant mechanism, the higher deposition rate of nanocatalysts leads to less mixing of fluids at the interface, which is due to less production of the low viscous fluid behind the interface. On the other hand, for DME dominant systems, higher deposition rates lead to more instability owing to less influence of nanocatalysts on the displacing fluid viscosity. We also introduce the effective penetration depth of nanocatalysts and show how they may lose their effectiveness due to their deposition in porous media. This is done through analyzing the global rate of the nanocatalytic reaction. This study provides new insight into the application of nanocatalysts in porous media.

\section{References}

[1] M.-C. Chang, H.-Y. Shu, W.-P. Hsieh, and M.-C. Wang, "Using nanoscale zero-valent iron for the remediation of polycyclic aromatic hydrocarbons contaminated soil," J. Air Waste Manag. Assoc., vol. 55, no. 8, pp. 1200-7, 2005.

[2] R. Hashemi, N. N. Nassar, and P. Pereira Almao, "Nanoparticle technology for heavy oil in-situ upgrading and recovery enhancement: Opportunities and challenges," Appl. Energy, vol. 133, pp. 374-387, 2014.

[3] N. Sabet, H. Hassanzadeh, and J. Abedi, "A new insight into the stability of variable viscosity diffusive boundary layers in porous media under gravity field," AIChE J., vol. 64, no. 3, pp. 1083-1094, 2018.

[4] C. T. Tan and G. M. Homsy, "Stability of miscible displacements in porous media: Rectilinear flow," Phys. Fluids, vol. 29, no. 11, p. 3549, 1986. 
[5] N. Sabet, H. Hassanzadeh, and J. Abedi, "Control of viscous fingering by nanoparticles," Phys. Rev. E, vol. 96, no. 6, 2017.

[6] C. T. Tan and G. M. Homsy, "Simulation of nonlinear viscous fingering in miscible displacement," Phys. Fluids, vol. 31, no. 6, p. 1330, 1988. 